\title{
CRESCIMENTO E NUTRIÇÃO MINERAL DE MUDAS DE CEDRO AUSTRALIANO (Toona ciliata) SOB OMISSÃO DE NUTRIENTES
}

\author{
Bruno da Silva Moretti ${ }^{1}$, Antonio Eduardo Furtini $\mathrm{Neto}^{2}$, Sheila Isabel do Carmo Pinto ${ }^{3}$, \\ Isabela Volpi Furtini ${ }^{4}$, Ciro Augusto de Souza Magalhães ${ }^{5}$
}

(recebido: 7 de dezembro de 2009; aceito: 30 de junho de 2011)

\begin{abstract}
RESUMO: Com o objetivo de se avaliar as exigências nutricionais e o efeito da omissão de nutrientes no crescimento de mudas de cedro australiano (Toona ciliata $\mathrm{M}$. Roem var. australis), foi realizado um experimento em casa de vegetação. Utilizou-se como substrato um Latossolo Vermelho Distroférrico, com baixa disponibilidade de nutrientes, utilizando-se 15 tratamentos, sob a técnica do elemento faltante. Foram aplicados dois tratamentos completos (um com fornecimento de N, P, K, S, B, Cu, Zn e aplicação de calcário e outro com o fornecimento de $\mathrm{N}, \mathrm{P}, \mathrm{K}, \mathrm{Ca}, \mathrm{Mg}, \mathrm{S}, \mathrm{B}, \mathrm{Cu}$ e Zn, sem a aplicação de calcário), além da omissão de cada nutriente (-N, -P, -K, -Ca, -Mg, -S, -B, -Cu e-Zn), um tratamento com omissão conjunta de B, Cu e Zn, um tratamento com aplicação apenas de calcário, um tratamento com aplicação de N, P, K, S, B, Cu e Zn, sem aplicação de calcário e uma testemunha absoluta (solo natural). Foram avaliadas as seguintes características: altura, diâmetro, matéria seca da parte aérea e das raízes, e teor de nutrientes na matéria seca da parte aérea, ao final de 150 dias. As plantas de cedro australiano apresentam elevada exigência nutricional, sendo que os nutrientes $\mathrm{P}, \mathrm{N}, \mathrm{S}, \mathrm{Ca}, \mathrm{K}, \mathrm{Mg}$ e $\mathrm{Cu}$, nessa ordem, foram limitantes ao crescimento das plantas. A omissão de B e Zn não afetou o crescimento das plantas. A aplicação de calcário foi essencial para o desenvolvimento das plantas de cedro australiano. O aparecimento dos primeiros sintomas de deficiência foi decorrente da omissão de: S, calcário e N.
\end{abstract}

Palavras-chave: Elemento faltante, fertilidade do solo, deficiência nutricional.

\section{GROWTH AND MINERAL NUTRITION IN SEEDLINGS OF AUSTRALIAN CEDAR (Toona ciliata) SUBJECTED TO NUTRIENT DEPRIVATION}

\begin{abstract}
In order to evaluate nutritional requirements and the effect of nutrient deprivation on the development of seedlings of Australian cedar (Toona ciliata M. Roem var. australis), a greenhouse experiment was conducted. The substrate used was a dystroferric red latosol with low nutrient availability, using 15 treatments and applying the missing element technique. The experiment included two complete treatments (one provided $N, P, K, S, B, C u, Z n$ with limestone while another provided $N, P, K, C a, M g, S$, $B, C u$ and $Z n$ without limestone), besides deprivation of each nutrient $(-N,-P,-K,-C a,-M g,-S,-B,-C u$ and $-Z n)$, one treatment with combined deprivation of $B, C u$ and $\mathrm{Zn}$, one treatment applying limestone only, one treatment applying $N, P, K, S, B, C u$ and $\mathrm{Zn}$, without limestone, and one absolute control treatment (natural soil). The following characteristics were evaluated: height, diameter, shoot dry matter and root dry matter, and nutrient content in the shoot dry matter after 150 days. Australian cedar plants have high nutritional requirements, and nutrients $P, N, S, C a, K, M g$ and $\mathrm{Cu}$, in that order, were found to be limiting factors to plant development. $B$ and $\mathrm{Zn}$ deprivation did not affect plant development. Limestone application was essential for the development of Australian cedar plants. Initial deficiency symptoms were found to be the result of $S$, limestone and $N$ deprivation.
\end{abstract}

Key words: Missing element, soil fertility, nutritional deficiency.

\section{INTRODUÇÃO}

No Brasil, os estudos silviculturais têm se voltado, quase que exclusivamente, para as espécies de rápido crescimento, como, por exemplo, as do gênero Eucalyptus e Pinus. Entretanto, outras espécies devem ser estudadas visando a uma maior diversificação no tocante à produção florestal. Uma espécie que tem se mostrado bastante promissora para esse fim é o cedro-australiano (Toona ciliata M. Roem var. australis).

O cedro australiano pertencente à família Meliaceae, é uma espécie originária das regiões tropicais da Austrália

\footnotetext{
${ }^{1}$ Agrônomo, Mestrando em Ciência do Solo - Departamento de Ciência do Solo - Universidade Federal de Lavras/UFLA - Cx.P. 3037 - $37200-000$ Lavras, MG - bsmoretti@hotmail.com

${ }^{2}$ Agrônomo, Professor Dr. em Solos e Nutrição de Plantas - Departamento de Ciência do Solo - Universidade Federal de Lavras/UFLA - Cx. P. 3037 37200-000 - Lavras, MG - afurtini@dcs.ufla.br

${ }^{3}$ Engenheira Florestal, Professora Dra. em Ciência do Solo - Instituto Federal Minas Gerais - Escola Agrotécnica Federal de Bambuí - Fazenda Varginha - Rodovia Bambuí-Medeiros, Km 05 - Cx. P. 05 - 38900-000 - Bambui, MG - sheilaicp@yahoo.com.br

${ }^{4}$ Agrônoma, Dra. em Genética e Melhoramento de Plantas - Departamento de Biologia - Universidade Federal de Lavras/UFLA - $37200-000$ Lavras, MG - isafurtini@yahoo.com.br

${ }^{5}$ Engenheiro Agrícola, Pesquisador Dr. em Ciência do Solo - Embrapa Roraima - Rodovia BR-174, Km 8 - Distrito Industrial - 69301-970 - Boa Vista, RR - ciroasm@yahoo.com.br
}

Cerne, Lavras, v. 17, n. 4, p. 453-463, out./dez. 2011 
que se adaptou muito bem ao Brasil, onde encontrou excelentes condições para o seu desenvolvimento, principalmente no sul da Bahia e em toda a Região Sudeste (PINHEIRO et al., 2003). Essa espécie apresenta rápido crescimento, chegando a atingir oito metros de altura e 15 $\mathrm{cm}$ de diâmetro com três anos de idade (PINHEIRO et al., 1994), proporcionando assim, um bom retorno financeiro em curto espaço de tempo, quando comparado aos cedros nativos e a outras essências florestais, inclusive o mogno (Swietenia macrophylla king).

A carência de estudos envolvendo a absorção de nutrientes e requerimentos nutricionais do cedroaustraliano, bem como envolvendo a sensibilidade a variadas condições de estresses químicos e físicos vêm sendo um entrave para que sua utilização se torne amplamente difundida.

Uma maneira rápida e econômica de se conhecer as exigências nutricionais de uma espécie é por meio da técnica do elemento faltante. Essa técnica envolve o crescimento de uma planta sob condições de campo ou casa de vegetação, em que é testado um tratamento completo (com todos os nutrientes necessários em doses adequadas) e uma série de tratamentos, nos quais é feita a omissão de um nutriente de cada vez. Normalmente, considera-se como deficiência severa quando a produção de massa seca cai a $40 \%$ em relação ao tratamento completo (SANCHES, 1981). Empregando-se a técnica do elemento faltante, pode-se verificar quais os nutrientes que se apresentam deficientes, a importância relativa dessa deficiência e a taxa de redução da fertilidade do solo quando se utilizam plantas que permitem cortes sucessivos (CHAMINADE, 1972). Segundo Malavolta (1980), ela representa uma referência semiquantitativa da necessidade de adubação. Vários trabalhos vêm sendo realizados com diferentes espécies utilizando-se essa técnica (SILVA et al., 2005, 2007; SOUZA et al., 2006; VENTURIN et al., 2005).

Conduziu-se o presente trabalho, com o objetivo de avaliar o efeito da omissão de nutrientes no crescimento, produção de matéria seca, teores de macro e micronutrientes e alterações morfológicas de mudas de cedro australiano.

\section{MATERIAL E MÉTODOS}

$\mathrm{O}$ experimento foi instalado em casa de vegetação no Departamento de Ciência do Solo da Universidade Federal de Lavras - MG (DCS/UFLA), usando-se como substrato um Latossolo Vermelho Distroférrico típico (LVdf), textura muito argilosa (EMPRESA BRASILEIRA
DE PESQUISA AGROPECUÁRIA - EMBRAPA, 1997), coletado em uma área atualmente cultivada com eucalipto. O clima da região foi classificado como $\mathrm{CWa}$, no sistema Köppen, com inverno seco e médias anuais de precipitação e temperatura de $1530 \mathrm{~mm}$ e $19,4^{\circ} \mathrm{C}$, respectivamente.

O solo utilizado no experimento foi caracterizado química (Tabela 1) e fisicamente, antes da instalação dos experimentos. O solo foi seco ao ar e passado em peneira de $2 \mathrm{~mm}$. Na fração TFSA foi realizada a análise granulométrica pelo método da pipeta (EMBRAPA, 1997), com uso de dispersante químico $\left(\mathrm{NaOH} 1 \mathrm{~mol} \mathrm{~L}^{-1}\right)$. A densidade de partículas foi determinada pelo método do balão volumétrico (BLAKE; HARTGE, 1986). Nessa mesma fração, foram determinados: carbono orgânico, complexo sortivo, $\mathrm{pH}$ em água (relação solo: solução 1:2,5) (EMBRAPA, 1997). Os micronutrientes $(\mathrm{Cu}, \mathrm{Fe}$, Mn e Zn) foram extraídos pelo Mehlich-1, e o boro por água quente. A disponibilidade de $\mathrm{P}$ foi avaliada também pela resina de troca iônica (RAIJ et al., 1986). Todas as análises foram realizadas nos laboratórios do DCS/UFLA.

Após a caracterização do solo, foram instalados os experimentos em vasos com capacidade para três $\mathrm{dm}^{3}$ (Tabela 2). Para os tratamentos que previam calagem, esta foi feita com aplicação de calcário dolomítico, visando a elevar a saturação por bases para 50\%. Após a aplicação do calcário, o solo foi incubado por um período de aproximadamente 30 dias, mantendo-se a umidade a $60 \%$ do volume total de poros (VTP), após o qual foram retiradas subamostras dos vasos para novas determinações químicas, as quais se encontram na Tabela 1. Posteriormente, foi transplantada uma muda de cedro australiano por vaso, proveniente de tubete e com idade aproximada de 60 dias e $21 \mathrm{~cm}$ de altura média. Nessa ocasião, o substrato foi lavado e retirado das mudas antes do transplantio.

A adubação básica de plantio nos diversos tratamentos, quando pertinente, constou de soluções nutritivas contendo $\left(\mathrm{mg} \mathrm{dm}^{-3}\right): 80$ de N, 200 de P, 150 de K, 50 de S, 75 de $\mathrm{Ca}, 15$ de $\mathrm{Mg}, 0,5$ de B, 1,5 de Cu e 5,0 de Zn (MALAVOLTA, 1980). As fontes dos nutrientes foram os sais p.a. $\mathrm{Ca}\left(\mathrm{H}_{2} \mathrm{PO}_{4}\right) \cdot 2 \mathrm{H}_{2} \mathrm{O}, \mathrm{KH}_{2} \mathrm{PO}_{4}, \mathrm{KNO}_{3}$, $\mathrm{MgSO}_{4} \cdot 7 \mathrm{H}_{2} \mathrm{O}, \mathrm{K}_{2} \mathrm{SO}_{4}, \mathrm{NH}_{4} \mathrm{H}_{2} \mathrm{PO}_{4}, \mathrm{H}_{3} \mathrm{PO}_{4}, \mathrm{Ca}\left(\mathrm{NO}_{3}\right)_{2} \cdot 4 \mathrm{H}_{2} \mathrm{O}$, $\mathrm{Mg}\left(\mathrm{NO}_{3}\right)_{2} \cdot 6 \mathrm{H}_{2} \mathrm{O},\left(\mathrm{NH}_{4}\right) \mathrm{SO}_{4}, \mathrm{CaSO}_{4} \cdot 2 \mathrm{H}_{2} \mathrm{O}, \mathrm{H}_{3} \mathrm{BO}_{3}, \mathrm{CuCl}_{2}$, $\mathrm{ZnCl}_{2}, \mathrm{NH}_{4} \mathrm{NO}_{3}$.

Os dados obtidos para as diferentes variáveis foram submetidos às análises de variância e as médias foram comparadas pelo teste de Scott-Knott a 5\%, utilizando-se o software estatístico Sisvar (FERREIRA, 2000).

Cerne, Lavras, v. 17, n. 4, p. 453-463, out./dez. 2011 


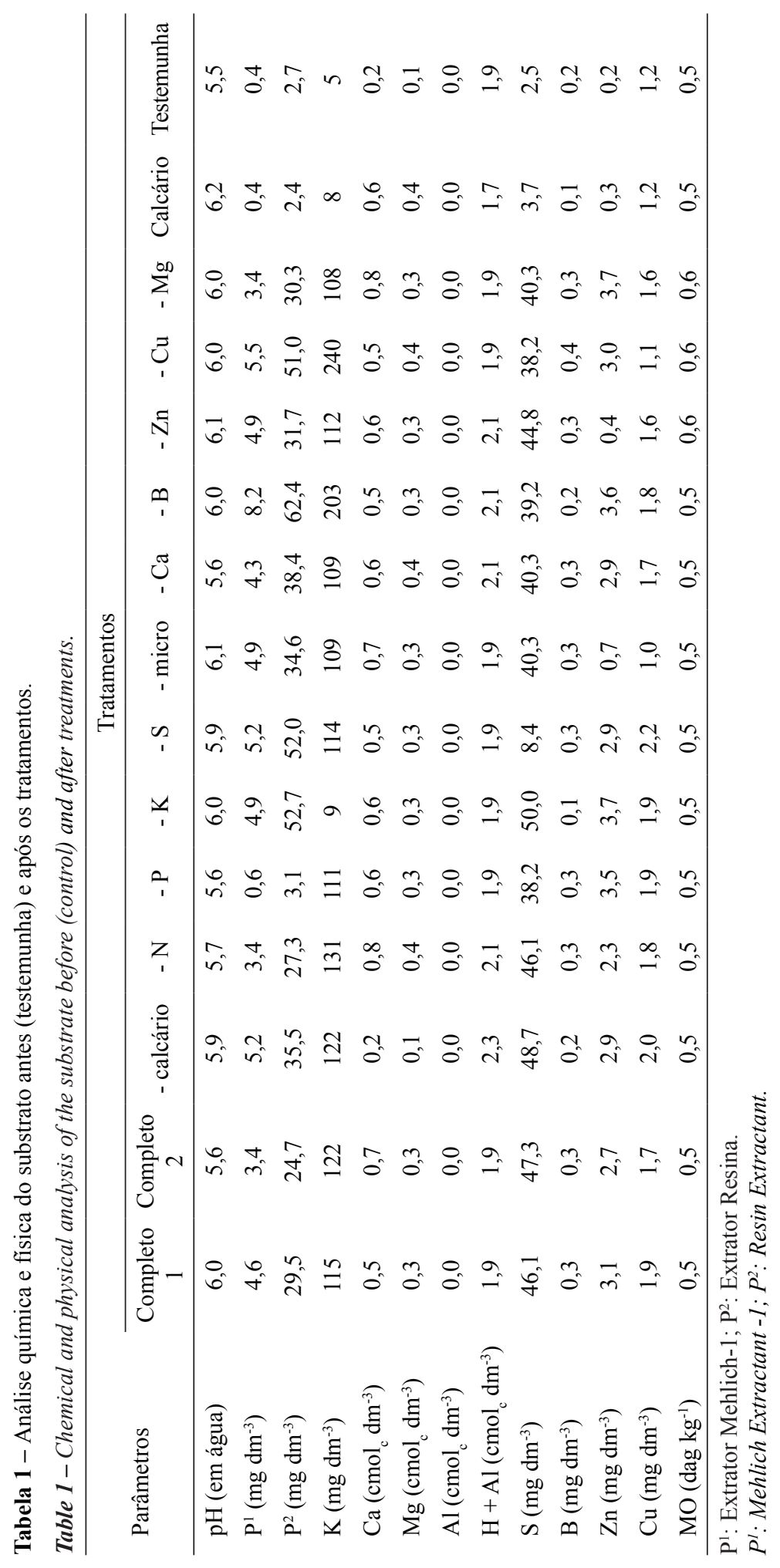

Cerne, Lavras, v. 17, n. 4, p. 453-463, out./dez. 2011 
Tabela 2 - Identificação e caracterização dos tratamentos.

Table 2 - Identification and characterization of treatments.

\begin{tabular}{|c|c|}
\hline Tratamento & Caracterização \\
\hline Comp. 1 & Aplicação de N, P, K, S, B, Cu, Zn + calcário \\
\hline Comp. 2 & Completo $1-$ calcário $+\mathrm{Ca}\left(\mathrm{PO}_{4}\right)$ e $\mathrm{Mg}\left(\mathrm{NO}_{3}\right)$ \\
\hline - Calc. & Completo 1 - Calcário \\
\hline$-\mathrm{N}$ & Completo 1- N \\
\hline$-\mathrm{P}$ & Completo 1 - P \\
\hline$-\mathrm{K}$ & Completo 1 - K \\
\hline$-\mathrm{S}$ & Completo 1 - S \\
\hline - Micro & Completo 1 - Micronutrientes \\
\hline$-\mathrm{Ca}$ & Completo 2 - $\mathrm{Ca}$ \\
\hline$-\mathrm{B}$ & Completo 1 - B \\
\hline$-\mathrm{Zn}$ & Completo 1 - Zn \\
\hline$-\mathrm{Cu}$ & Completo $1-\mathrm{Cu}$ \\
\hline$-\mathrm{Mg}$ & Completo 2 - Mg \\
\hline Calc. & Solo Natural + calcário \\
\hline Test. & Solo Natural \\
\hline
\end{tabular}

Durante a condução do experimento, a umidade foi mantida em $60 \%$ do VTP, de acordo com Freire et al. (1980), efetuando-se pesagens diárias dos vasos, e complementação do peso com água deionizada. Foram avaliados a altura das plantas e diâmetro de caule no dia do transplantio (29/03/08) e a cada 30 dias após o transplantio.

Os valores de alturas das plantas foram obtidos medindo do nível do solo ao meristema apical e para o diâmetro de caule as medidas foram tomadas no nível do solo.

Durante todo o período experimental, avaliaram-se os sintomas visuais das plantas, quando o nível exigido para o seu desenvolvimento está abaixo do considerado suficiente. As descrições de deficiência nutricional de um determinado nutriente são relatadas por Faquin (2005) e Malavolta et al. (1997) e foram tomadas como base para o diagnóstico visual.

Aos 150 dias após o transplantio, foram coletadas as folhas fisiologicamente maduras, a parte aérea e as raízes de todas as plantas, juntamente com as folhas que senesceram e caíram durante a condução do experimento. Esses materiais foram secos em estufa de circulação de ar forçada a $65^{\circ} \mathrm{C}$. Após seco, os materiais foram triturados em moinho tipo Willey e, em seguida, foram efetuadas as análises químicas para a determinação dos teores totais de nutrientes (MALAVOLTA et al., 1997) e quantidade total de matéria seca da parte aérea (MSPA), separando-a em matéria seca do limbo foliar (MSF), matéria seca do caule (MSC) e matéria seca do pecíolo (MSP), e matéria seca de raiz (MSR) do cedro australiano. Foi obtido o crescimento relativo $(\mathrm{CR})$ por meio da seguinte expressão: $\mathrm{CR}(\%)=$ $($ MSON/MSTC) $\times 100$, onde: $\mathrm{MSON}=$ massa seca total obtida em cada omissão de nutriente e MSTC = massa seca total obtida no tratamento completo.

O delineamento utilizado foi o inteiramente casualizado (DIC), com 15 tratamentos e cinco repetições. O experimento constou de 75 vasos plásticos de três $\mathrm{dm}^{3}$ de solo, cada vaso correspondendo a uma parcela experimental.

\section{RESULTADOS E DISCUSSÃO}

\subsection{Crescimento das plantas}

Ao término do período experimental, todos os tratamentos apresentaram altura e diâmetro de plantas, iguais ou inferiores ao tratamento completo 1 (Tabela 3).

Os nutrientes $\mathrm{N}, \mathrm{P}, \mathrm{K}, \mathrm{S}$ e Mg além do calcário foram os que mais limitaram o crescimento inicial das plantas de cedro australiano. A altura das plantas do tratamento com omissão de $\mathrm{N}$ e $\mathrm{K}$, até a $3^{\mathrm{a}}$ avaliação, foi pouco afetada. A partir daí, esses nutrientes mostraram-se limitantes ao crescimento em altura das plantas. Os tratamentos completo 1 e completo 2 apresentaram comportamento diferente, o tratamento completo 1 foi sempre superior ao completo 2. Uma explicação para esse fato é que o tratamento completo 2 , por não ter tido o fornecimento de calcário, não teve o seu $\mathrm{pH}$ corrigido e, consequentemente, a disponibilidade de alguns nutrientes ficou comprometida. Já, o tratamento com omissão de Ca, em todas as avaliações, apresentou altura semelhante ao tratamento completo 1 . A testemunha sempre apresentou menor crescimento em altura, justificando a importância da aplicação de nutrientes para o desenvolvimento das plantas de cedro australiano.

Assim como ocorreu com o crescimento em altura das plantas, os tratamentos com omissão de calcário e com aplicação apenas de calcário, prejudicou o crescimento em diâmetro do caule.

Dentre os micronutrientes omitidos, o $\mathrm{Cu}$ foi o que mais limitou o crescimento inicial em altura das plantas de cedro australiano até a $3^{\text {a }}$ avaliação, nas duas seguintes, esse tratamento foi superior estatisticamente. Já que nas demais avaliações não diferiram do tratamento completo 1. Quanto ao B e Zn infere-se, em princípio, que a espécie não é muito exigente nesses nutrientes, pelo menos nessa fase de crescimento.

Cerne, Lavras, v. 17, n. 4, p. 453-463, out./dez. 2011 
Tabela 3 - Altura (A) e diâmetro (D) de plantas de cedro australiano em cinco avaliações, submetidas aos diferentes tratamentos (Trat) ${ }^{1}$.

Table 3 - Height (A) and diameter (D) of Australian cedar plants in five evaluations, subjected to different treatments (Treat) ${ }^{\text {. }}$.

\begin{tabular}{|c|c|c|c|c|c|c|c|c|c|c|c|c|c|c|c|c|c|c|c|c|}
\hline \multirow{3}{*}{ Trat } & \multicolumn{4}{|c|}{$30 \mathrm{DAT}$} & \multicolumn{4}{|c|}{$60 \mathrm{DAT}$} & \multicolumn{4}{|c|}{$90 \mathrm{DAT}$} & \multicolumn{4}{|c|}{$120 \mathrm{DAT}$} & \multicolumn{4}{|c|}{$150 \mathrm{DAT}$} \\
\hline & $\mathrm{A}$ & & $\mathrm{D}$ & & $\mathrm{A}$ & & $\mathrm{D}$ & & $\mathrm{A}$ & & $\mathrm{D}$ & & $\mathrm{A}$ & & $\mathrm{D}$ & & $\mathrm{A}$ & & $\mathrm{D}$ & \\
\hline & $\mathrm{cm}$ & & $\mathrm{mm}$ & & $\mathrm{cm}$ & & $\mathrm{mm}$ & & $\mathrm{cm}$ & & $\mathrm{mm}$ & & $\mathrm{cm}$ & & $\mathrm{mm}$ & & $\mathrm{cm}$ & & $\mathrm{mm}$ & \\
\hline Comp. 1 & 21,8 & $\mathrm{a}$ & 6,2 & $\mathrm{a}$ & 23,0 & $\mathrm{a}$ & 7,7 & $\mathrm{a}$ & 23,9 & $\mathrm{a}$ & 8,2 & $\mathrm{a}$ & 27,9 & $\mathrm{a}$ & 9,6 & $\mathrm{a}$ & 28,5 & $\mathrm{a}$ & 11,0 & $\mathrm{a}$ \\
\hline Comp. 2 & 20,5 & $\mathrm{~b}$ & 6,4 & a & 21,1 & $\mathrm{~b}$ & 7,3 & $\mathrm{a}$ & 21,8 & $\mathrm{~b}$ & 7,7 & $\mathrm{a}$ & 24,7 & $\mathrm{~b}$ & 9,2 & $\mathrm{a}$ & 25,7 & $\mathrm{~b}$ & 9,8 & $\mathrm{~b}$ \\
\hline - Calc. & 20,5 & $\mathrm{~b}$ & 6,7 & $\mathrm{a}$ & 21,2 & $\mathrm{~b}$ & 7,0 & $\mathrm{a}$ & 21,5 & $\mathrm{~b}$ & 7,4 & $\mathrm{~b}$ & 22,5 & $\mathrm{~b}$ & 8,1 & $\mathrm{~b}$ & 23,1 & $\mathrm{~b}$ & 9,1 & $\mathrm{~b}$ \\
\hline$-\mathrm{N}$ & 22,2 & $\mathrm{a}$ & 6,7 & a & 22,9 & a & 7,7 & a & 23,6 & $\mathrm{a}$ & 7,9 & $\mathrm{a}$ & 23,9 & $\mathrm{~b}$ & 9,2 & $\mathrm{a}$ & 24,4 & $\mathrm{~b}$ & 9,9 & $\mathrm{~b}$ \\
\hline$-P$ & 21,0 & $\mathrm{~b}$ & 5,9 & $\mathrm{a}$ & 21,6 & $\mathrm{~b}$ & 6,6 & $\mathrm{a}$ & 22,6 & $\mathrm{a}$ & 7,0 & $\mathrm{~b}$ & 22,7 & $\mathrm{~b}$ & 8,6 & $\mathrm{~b}$ & 23,2 & $\mathrm{~b}$ & 9,3 & $\mathrm{~b}$ \\
\hline$-\mathrm{K}$ & 22,2 & $\mathrm{a}$ & 6,4 & $\mathrm{a}$ & 23,0 & $\mathrm{a}$ & 7,0 & $\mathrm{a}$ & 23,6 & $\mathrm{a}$ & 7,6 & $\mathrm{a}$ & 24,6 & $\mathrm{~b}$ & 9,0 & $\mathrm{a}$ & 25,4 & $\mathrm{~b}$ & 9,4 & $\mathrm{~b}$ \\
\hline$-\mathrm{S}$ & 20,6 & $\mathrm{~b}$ & 6,4 & $\mathrm{a}$ & 21,1 & $\mathrm{~b}$ & 6,9 & $\mathrm{a}$ & 21,7 & $\mathrm{~b}$ & 7,4 & $\mathrm{~b}$ & 23,9 & $\mathrm{~b}$ & 7,8 & $\mathrm{~b}$ & 25,2 & $\mathrm{~b}$ & 8,8 & $\mathrm{~b}$ \\
\hline$-\mathrm{Ca}$ & 21,4 & $\mathrm{a}$ & 6,3 & $\mathrm{a}$ & 21,8 & $\mathrm{~b}$ & 7,4 & $\mathrm{a}$ & 22,9 & $\mathrm{a}$ & 7,7 & $\mathrm{a}$ & 25,9 & $\mathrm{a}$ & 9,2 & $\mathrm{a}$ & 28,7 & $\mathrm{a}$ & 10,2 & $\mathrm{a}$ \\
\hline$-\mathrm{Mg}$ & 20,9 & $\mathrm{~b}$ & 6,7 & $\mathrm{a}$ & 21,5 & $\mathrm{~b}$ & 8,3 & $\mathrm{a}$ & 22,2 & $\mathrm{~b}$ & 8,8 & $\mathrm{a}$ & 23,7 & $\mathrm{~b}$ & 10,4 & $\mathrm{a}$ & 24,7 & $\mathrm{~b}$ & 11,2 & $\mathrm{a}$ \\
\hline - Micro & 21,1 & $\mathrm{~b}$ & 7,3 & $\mathrm{a}$ & 21,8 & $\mathrm{~b}$ & 7,8 & $\mathrm{a}$ & 22,4 & $\mathrm{a}$ & 8,1 & $\mathrm{a}$ & 25,5 & $\mathrm{a}$ & 9,8 & $\mathrm{a}$ & 26,6 & $\mathrm{a}$ & 11,3 & $\mathrm{a}$ \\
\hline$-B$ & 22,2 & $\mathrm{a}$ & 6,6 & $\mathrm{a}$ & 22,9 & $\mathrm{a}$ & 7,3 & $\mathrm{a}$ & 23,7 & $\mathrm{a}$ & 7,7 & $\mathrm{a}$ & 28,7 & $\mathrm{a}$ & 10,2 & $\mathrm{a}$ & 30,4 & $\mathrm{a}$ & 11,1 & $\mathrm{a}$ \\
\hline$-Z n$ & 21,9 & $\mathrm{a}$ & 7,0 & $\mathrm{a}$ & 22,6 & $\mathrm{a}$ & 7,8 & $\mathrm{a}$ & 23,0 & $\mathrm{a}$ & 8,2 & $\mathrm{a}$ & 29,3 & $\mathrm{a}$ & 10,4 & $\mathrm{a}$ & 31,0 & $\mathrm{a}$ & 11,2 & $\mathrm{a}$ \\
\hline$-\mathrm{Cu}$ & 20,6 & $\mathrm{~b}$ & 7,3 & $\mathrm{a}$ & 21,1 & $\mathrm{~b}$ & 8,2 & $\mathrm{a}$ & 21,7 & $\mathrm{~b}$ & 8,8 & $\mathrm{a}$ & 25,8 & $\mathrm{a}$ & 10,4 & $\mathrm{a}$ & 26,8 & $\mathrm{a}$ & 11,8 & $\mathrm{a}$ \\
\hline Calc. & 20,5 & $\mathrm{~b}$ & 7,0 & $\mathrm{a}$ & 20,9 & $\mathrm{~b}$ & 7,2 & $\mathrm{a}$ & 21,4 & $\mathrm{~b}$ & 7,2 & $\mathrm{~b}$ & 21,8 & $\mathrm{~b}$ & 7,60 & $\mathrm{~b}$ & 21,7 & $\mathrm{~b}$ & 8,60 & $\mathrm{~b}$ \\
\hline Test. & 19,0 & $\mathrm{~b}$ & 5,8 & $\mathrm{a}$ & 19,0 & $\mathrm{c}$ & 5,8 & $\mathrm{a}$ & 19,2 & $\mathrm{c}$ & 5,9 & $\mathrm{c}$ & 19,3 & $\mathrm{~b}$ & 6,30 & $\mathrm{c}$ & 19,8 & $\mathrm{~b}$ & 7,0 & $\mathrm{c}$ \\
\hline $\mathrm{CV}(\%)$ & 5,7 & & 11,7 & & 6,0 & & 12,6 & & 6,05 & & 10,2 & & 14,1 & & 11,9 & & 14,0 & & 11,23 & \\
\hline
\end{tabular}

${ }^{1}$ As médias seguidas da mesma letra nas colunas, pertencem ao mesmo grupo, pelo teste de Scott-Knott, a 5\% de probabilidade.

Os tratamentos com omissão de calcário e com aplicação apenas de calcário não diferiram entre si, e resultaram em menor crescimento em altura das plantas, quando comparados ao tratamento completo 1. Esse fato ressalta a importância da realização das práticas conjuntas de calagem e adubação, pois, na ausência de uma dessas práticas, o desenvolvimento das plantas ficou comprometido.

O crescimento em diâmetro do caule até a segunda avaliação não diferiu estatisticamente entre os tratamentos (Tabela 3). Porém, a partir da terceira avaliação, verificouse que os macronutrientes mais limitantes foram o $\mathrm{P}$ e $\mathrm{S}$. Na última avaliação, observou-se que, além do $\mathrm{P}$ e $\mathrm{S}$, os nutrientes $\mathrm{N}$ e $\mathrm{K}$ também afetaram o crescimento em diâmetro do caule das plantas de cedro australiano.

Com relação à omissão de micronutrientes, durante o período avaliado, não houve diferença no crescimento em diâmetro quando esses tratamentos foram comparados com o tratamento completo 1. É importante enfatizar que a testemunha apresentou o pior crescimento a partir da terceira avaliação, reforçando a importância do fornecimento de nutrientes à espécie. Como o desenvolvimento em diâmetro das plantas tem forte influência da base genética de cada espécie, provavelmente essa variável ainda não teria sido afetada pelos tratamentos, durante o período de condução do experimento, em torno de 150 dias. Entretanto, como ocorreu efeito dos tratamentos em várias outras variáveis de crescimento, espera-se que com a idade, também o diâmetro do caule possa ser influenciado pela omissão de nutrientes.

$\mathrm{Na}$ fase de mudas, o experimento foi conduzido durante 150 dias, período em que o crescimento em altura não foi significativo. Assim, avaliaram-se diversos parâmetros, descritos a seguir, para analisar o efeito de cada tratamento.

\subsection{Produção de matéria seca}

Conforme pode ser observado na Tabela 4, as plantas dos tratamentos com omissão conjunta de micronutrientes e de B e Zn não diferiram em produção de matéria seca de folhas (MSF), matéria seca dos pecíolos (MSP) e matéria seca da parte aérea (MSPA) das plantas dos tratamentos completo 1 e completo 2 . Nessa tabela, percebe-se que os tratamentos com omissão de micronutrientes, omissão de B e omissão de Zn mostraram maior produção para a

Cerne, Lavras, v. 17, n. 4, p. 453-463, out./dez. 2011 
Tabela 4 - Matéria seca de folhas (MSF), dos pecíolos (MSP), do caule (MSC), da parte aérea (MSPA) e das raízes (MSR) de plantas de cedro australiano, aos 150 dias após transplante, submetidas aos diferentes tratamentos ${ }^{1}$.

Table 4 - Leaf dry matter (MSF), petiole dry matter (MSP), stem dry matter (MSC), shoot dry matter (MSPA) and root dry matter (MSR) in Australian cedar plants, 150 days after transplantation, subjected to different treatments ${ }^{\text {. }}$.

\begin{tabular}{|c|c|c|c|c|c|c|c|c|c|c|c|}
\hline \multirow{2}{*}{$\begin{array}{l}\text { Tratamento } \\
\text { Completo } 1\end{array}$} & \multicolumn{2}{|l|}{ MSF } & \multicolumn{2}{|c|}{ MSP } & \multicolumn{2}{|c|}{ MSC } & \multicolumn{2}{|c|}{ MSPA } & \multicolumn{2}{|l|}{ MSR } & MSR/MSPA \\
\hline & 3,49 & $\mathrm{a}$ & 0,67 & $\mathrm{a}$ & 3,55 & $\mathrm{~b}$ & 7,72 & $\mathrm{a}$ & 11,06 & $\mathrm{a}$ & $1,43 \quad b$ \\
\hline Completo 2 & 3,07 & $\mathrm{a}$ & 0,73 & $\mathrm{a}$ & 3,14 & $\mathrm{~b}$ & 6,96 & $\mathrm{a}$ & 9,57 & $\mathrm{a}$ & $1,42 \quad b$ \\
\hline - Calcário & 2,48 & $\mathrm{~b}$ & 0,46 & $\mathrm{~b}$ & 2,43 & $\mathrm{c}$ & 5,39 & $\mathrm{~b}$ & 5,67 & $\mathrm{~b}$ & $1,09 \quad b$ \\
\hline$-\mathrm{N}$ & 1,05 & $\mathrm{c}$ & 0,33 & $\mathrm{c}$ & 2,97 & $\mathrm{~b}$ & 4,36 & $\mathrm{~b}$ & 8,79 & $\mathrm{a}$ & $2,00 \mathrm{a}$ \\
\hline$-\mathrm{P}$ & 1,45 & $\mathrm{c}$ & 0,23 & $\mathrm{c}$ & 2,40 & $\mathrm{c}$ & 4,01 & $\mathrm{~b}$ & 7,51 & $\mathrm{a}$ & $1,92 \mathrm{a}$ \\
\hline$-\mathrm{K}$ & 2,09 & $\mathrm{~b}$ & 0,30 & $\mathrm{c}$ & 2,99 & $\mathrm{~b}$ & 5,38 & $\mathrm{~b}$ & 7,72 & $\mathrm{a}$ & $1,42 \quad b$ \\
\hline$-\mathrm{S}$ & 2,26 & $\mathrm{~b}$ & 0,46 & $\mathrm{~b}$ & 1,94 & d & 4,67 & $\mathrm{~b}$ & 4,27 & $\mathrm{~b}$ & $0,93 \mathrm{~b}$ \\
\hline - Micronutrientes & 3,02 & $\mathrm{a}$ & 0,70 & $\mathrm{a}$ & 4,05 & $\mathrm{a}$ & 7,78 & $\mathrm{a}$ & 9,11 & $\mathrm{a}$ & $1,21 \quad b$ \\
\hline$-\mathrm{Ca}$ & 1,64 & $\mathrm{c}$ & 0,49 & $\mathrm{~b}$ & 2,72 & $\mathrm{c}$ & 4,87 & $\mathrm{~b}$ & 8,04 & $\mathrm{a}$ & $1,74 \mathrm{a}$ \\
\hline$-\mathrm{B}$ & 2,93 & $\mathrm{a}$ & 0,61 & $\mathrm{a}$ & 4,40 & $\mathrm{a}$ & 7,95 & $\mathrm{a}$ & 11,42 & $\mathrm{a}$ & $1,46 \quad b$ \\
\hline$-\mathrm{Zn}$ & 2,98 & $\mathrm{a}$ & 0,63 & $\mathrm{a}$ & 4,11 & $\mathrm{a}$ & 7,73 & $\mathrm{a}$ & 9,78 & $\mathrm{a}$ & $1,26 \mathrm{~b}$ \\
\hline$-\mathrm{Cu}$ & 2,30 & $\mathrm{~b}$ & 0,50 & $\mathrm{~b}$ & 3,48 & $\mathrm{~b}$ & 6,30 & $\mathrm{a}$ & 9,88 & $\mathrm{a}$ & 1,61 a \\
\hline$-\mathrm{Mg}$ & 1,95 & $\mathrm{~b}$ & 0,41 & $\mathrm{~b}$ & 3,38 & $\mathrm{~b}$ & 5,75 & $\mathrm{~b}$ & 10,43 & $\mathrm{a}$ & $1,81 \mathrm{a}$ \\
\hline Calcário & 0,77 & $\mathrm{c}$ & 0,10 & $\mathrm{c}$ & 1,70 & d & 2,59 & $\mathrm{c}$ & 4,34 & $\mathrm{~b}$ & $1,69 \mathrm{a}$ \\
\hline Testemunha & 0,53 & $\mathrm{c}$ & 0,11 & $\mathrm{c}$ & 1,32 & d & 1,97 & $\mathrm{c}$ & 2,59 & $\mathrm{~b}$ & $1,30 \quad \mathrm{~b}$ \\
\hline $\mathrm{CV}(\%)$ & 33,46 & & 38,8 & & 23,81 & & 22,23 & & 27,88 & & 25,95 \\
\hline
\end{tabular}

${ }^{1}$ As média seguidas da mesma letra nas colunas, pertencem ao mesmo grupo, pelo teste de Scott-Knott, a 5\% de probabilidade.

matéria seca do caule (MSC). O micronutriente cuja omissão foi mais limitante foi o $\mathrm{Cu}$. Esses resultados relatam, em princípio, uma menor exigência do cedro australiano pelos micronutrientes omitidos, principalmente B e Zn. De maneira geral, observa-se que as omissões de macronutrientes prejudicaram a produção de MSF, MSP, MSC e MSPA. Já, para matéria seca de raízes (MSR), as omissões de calcário e de enxofre foram as mais limitantes ao seu desenvolvimento.

Na Figura 1, é apresentado o crescimento relativo (CR) em produção de matéria seca. Considerou-se o tratamento completo $1 \mathrm{com}$ crescimento relativo igual a $100 \%$. Verifica-se que os tratamentos com omissão de micronutrientes, B e Zn foram semelhantes ao tratamento completo 1 para MSPA. A omissão dos demais nutrientes afetou a produção de MSPA das plantas de cedro australiano, sendo mais acentuadas as omissões de N, P, S e Ca. Em relação aos micronutrientes, a produção de matéria seca da parte aérea das plantas de cedro australiano foi afetada somente pela omissão de $\mathrm{Cu}$. Assim, a sequência de exigência nutricional apresentada pelas mudas de cedro australiano em relação ao tratamento completo, considerando a produção de matéria seca da parte aérea em ordem decrescente foi: $\mathrm{P}>\mathrm{N}>\mathrm{S}>\mathrm{Ca}>\mathrm{K}>\mathrm{Mg}>\mathrm{Cu}>\mathrm{Zn}>\mathrm{B}$.

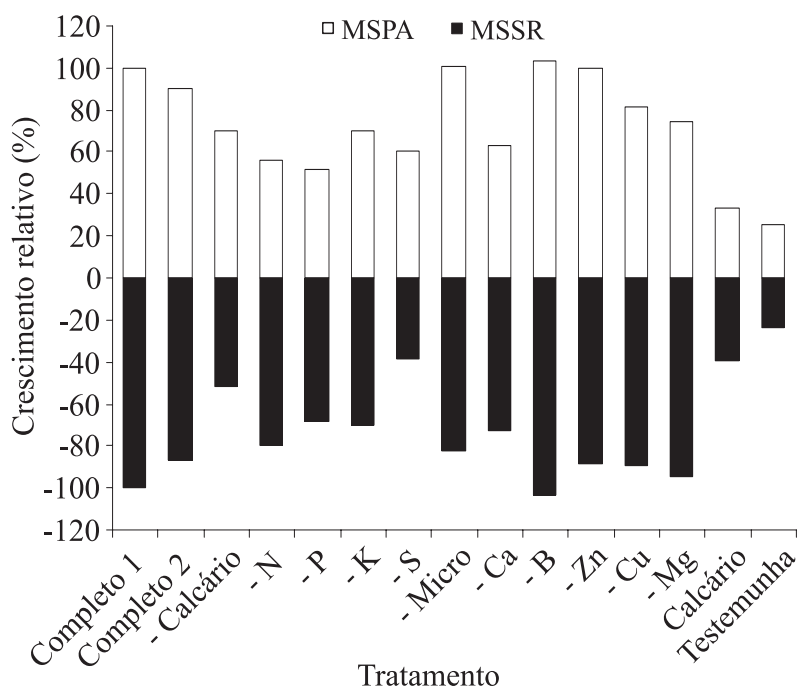

Figura 1 - Crescimento relativo em matéria seca da parte aérea (MSPA) e do sistema radicular (MSSR) de plantas de Cedro Australiano, submetidas a diferentes tratamentos.

Figure 1-Relative growth in shoot dry matter (MSPA) and root system dry matter (MSSR) in Australian cedar plants, subjected to different treatments.

Cerne, Lavras, v. 17, n. 4, p. 453-463, out./dez. 2011 
O sistema radicular do cedro australiano também foi significativamente afetado pela omissão de calcário, $\mathrm{S}$ além dos tratamentos "calcário" e "testemunha".

Os tratamentos que obtiveram as maiores relações MSR/MSPA foram aqueles com omissão de $\mathrm{N}, \mathrm{P}, \mathrm{Ca}, \mathrm{Mg}$ e $\mathrm{Cu}$ (Tabela 4). Já, os tratamentos que apresentaram os menores valores de MSR/MSPA foram aqueles em que não foram fornecidos $\mathrm{S}$, calcário e micronutrientes, além dos tratamentos completo 1 e completo 2. Em ambientes com baixa fertilidade natural, a relação R/PA é maior, pois a planta aumenta o volume de solo explorado como forma de maximizar a retirada de nutrientes nessas condições (CLARKSON, 1985). Marques et al. (2004) para o paricá (Schizolobium amazonicum) e Souza et al. (2006) para o ipêroxo (Tabebuia impetiginosa), também encontraram valores dessa relação maior que 1, embora Venturin et al. (1999) para o angico-amarelo (Peltophorum Dubium (Spreng.)Taub.), Locatelli et al. (2007) para o cedro rosa (Cedrela odorata L.) e Silva et al. (2007) para o mogno (Swietenia macrophylla king) tenham encontrado relações R/PA menores que 1, demonstrando, assim, que existem diferenças entre essas espécies em explorar o solo na busca pelos nutrientes.

\subsection{Teores de macronutrientes e micronutrientes no caule, nas folhas e nos pecíolos}

Os teores dos nutrientes na matéria seca da parte aérea (MSPA) do cedro australiano correspondentes a cada tratamento são apresentados na Tabela 5. De maneira geral, os menores teores de nutrientes na MSPA das plantas de cedro australiano foram observados nos tratamentos onde houve somente aplicação de calcário, na testemunha e na omissão de cada nutriente.

A absorção de $\mathrm{N}$ foi favorecida pelas omissões de $\mathrm{S}$ e K. O teor de $\mathrm{N}$ alcançado na MSPA pelo tratamento completo $\left(18,8 \mathrm{~g} \mathrm{~kg}^{-1}\right)$ foi superior aos encontrados em angico-amarelo (Peltophorum Dubium (Spreng.) Taub.) por Venturin et al. (1999), que relataram teores de $16,2 \mathrm{~g} \mathrm{~kg}^{-1}$ de N.

A omissão de $\mathrm{S}$ favoreceu também a absorção de $\mathrm{P}$ pelas plantas. $\mathrm{O}$ teor de $\mathrm{P}$ na MSPA, no tratamento 1 obtido no presente trabalho $\left(1,4 \mathrm{~g} \mathrm{~kg}^{-1}\right)$ foi superior ao relatado por Venturin et al. (1999), que encontraram teores de $0,76 \mathrm{~g} \mathrm{~kg}^{-1}$ de $\mathrm{P}$ em angico-amarelo (Peltophorum dubium (Spreng.) Taub.).

Tabela 5 - Teores de macronutrientes $\left(\mathrm{g} \mathrm{kg}^{-1}\right)$ e de micronutrientes $\left(\mathrm{mg} \mathrm{kg}^{-1}\right)$ na matéria seca da parte aérea do cedro australiano, em função dos tratamentos ${ }^{1}$.

Table 5-Macronutrient $\left(\mathrm{g} \mathrm{kg}^{-1}\right)$ and micronutrient $\left(\mathrm{mg} \mathrm{kg}^{-1}\right)$ contents in the shoot dry matter of Australian cedar plants, as a function of each treatment ${ }^{l}$.

\begin{tabular}{|c|c|c|c|c|c|c|c|c|c|c|c|c|c|c|c|c|c|c|}
\hline Trat. & $\mathrm{N}$ & & $\mathrm{P}$ & & $\mathrm{K}$ & & $\mathrm{Ca}$ & & $\mathrm{M}$ & & $\mathrm{S}$ & & B & & \multicolumn{2}{|c|}{$\mathrm{Cu}$} & \multicolumn{2}{|l|}{$\mathrm{Zn}$} \\
\hline Comp1 & 18,8 & $\mathrm{a}$ & 1,4 & $\mathrm{c}$ & 14,2 & $\mathrm{~b}$ & 10,5 & & 2,5 & $\mathrm{c}$ & 1,8 & $\mathrm{a}$ & 32,6 & $\mathrm{a}$ & 9,5 & $\mathrm{a}$ & 39,9 & $\mathrm{~b}$ \\
\hline Comp2 & 16,8 & $\mathrm{~b}$ & 1,3 & $\mathrm{c}$ & 15,5 & $\mathrm{a}$ & 9,8 & $\mathrm{~b}$ & 2,4 & $\mathrm{c}$ & 1,6 & $\mathrm{a}$ & 27,5 & $\mathrm{a}$ & 7,5 & $\mathrm{~b}$ & 49,2 & $\mathrm{a}$ \\
\hline - Calc. & 16,2 & $\mathrm{~b}$ & 1,7 & $\mathrm{~b}$ & 17,6 & $\mathrm{a}$ & 7,5 & $\mathrm{~b}$ & 1,4 & $\mathrm{~d}$ & 1,4 & $\mathrm{a}$ & 26,6 & $\mathrm{a}$ & 9,8 & $\mathrm{a}$ & 47,7 & $\mathrm{a}$ \\
\hline$-\mathrm{N}$ & 8,9 & $\mathrm{c}$ & 0,8 & $\mathrm{~d}$ & 14,6 & $\mathrm{~b}$ & 16,5 & $\mathrm{a}$ & 3,1 & $\mathrm{~b}$ & 1,2 & $\mathrm{a}$ & 30,5 & $\mathrm{a}$ & 6,7 & $\mathrm{~b}$ & 36,8 & $\mathrm{~b}$ \\
\hline$-\mathrm{P}$ & 17,6 & $\mathrm{~b}$ & 0,8 & $\mathrm{~d}$ & 12,9 & $\mathrm{~b}$ & 13,4 & $\mathrm{a}$ & 3,4 & $\mathrm{~b}$ & 1,3 & $\mathrm{a}$ & 34,1 & $\mathrm{a}$ & 6,7 & $\mathrm{~b}$ & 47,0 & $\mathrm{a}$ \\
\hline$-\mathrm{K}$ & 22,3 & $\mathrm{a}$ & 1,3 & $\mathrm{c}$ & 6,7 & $\mathrm{~d}$ & 17,6 & $\mathrm{a}$ & 4,8 & $\mathrm{a}$ & 1,6 & $\mathrm{a}$ & 28,4 & $\mathrm{a}$ & 8,3 & $\mathrm{a}$ & 59,3 & $\mathrm{a}$ \\
\hline$-\mathrm{S}$ & 21,5 & $\mathrm{a}$ & 2,3 & $\mathrm{a}$ & 15,2 & $\mathrm{~b}$ & 15,7 & $\mathrm{a}$ & 3,0 & $\mathrm{~b}$ & 0,8 & $\mathrm{~b}$ & 19,5 & $\mathrm{~b}$ & 9,9 & $\mathrm{a}$ & 42,1 & $\mathrm{~b}$ \\
\hline - Micr & 17,1 & $\mathrm{~b}$ & 1,5 & $\mathrm{~b}$ & 14,9 & $\mathrm{~b}$ & 11,7 & $\mathrm{~b}$ & 2,7 & $\mathrm{c}$ & 1,2 & $\mathrm{a}$ & 22,7 & $\mathrm{~b}$ & 8,3 & $\mathrm{a}$ & 33,0 & $\mathrm{~b}$ \\
\hline$-\mathrm{Ca}$ & 19,4 & $\mathrm{a}$ & 1,6 & $\mathrm{~b}$ & 16,2 & $\mathrm{a}$ & 10,9 & $\mathrm{~b}$ & 3,3 & $\mathrm{~b}$ & 1,7 & $\mathrm{a}$ & 22,8 & $\mathrm{~b}$ & 9,7 & $\mathrm{a}$ & 39,9 & $\mathrm{~b}$ \\
\hline$-\mathrm{B}$ & 15,2 & $\mathrm{~b}$ & 1,4 & $\mathrm{c}$ & 17,0 & $\mathrm{a}$ & 8,0 & $\mathrm{~b}$ & 2,3 & $\mathrm{c}$ & 1,2 & $\mathrm{a}$ & 17,7 & $\mathrm{~b}$ & 8,3 & a & 35,8 & $\mathrm{~b}$ \\
\hline$-\mathrm{Zn}$ & 18,8 & $\mathrm{a}$ & 1,7 & $\mathrm{~b}$ & 16,5 & $\mathrm{a}$ & 10,2 & $\mathrm{~b}$ & 3,1 & $\mathrm{~b}$ & 1,3 & $\mathrm{a}$ & 24,0 & $\mathrm{~b}$ & 10,1 & $\mathrm{a}$ & 24,9 & $\mathrm{~b}$ \\
\hline$-\mathrm{Cu}$ & 20,4 & $\mathrm{a}$ & 1,8 & $\mathrm{~b}$ & 15,7 & $\mathrm{a}$ & 10,1 & & 3,2 & $\mathrm{~b}$ & 0,9 & $\mathrm{~b}$ & 27,6 & $\mathrm{a}$ & 8,7 & a & 46,6 & $\mathrm{a}$ \\
\hline$-\mathrm{Mg}$ & 15,2 & $\mathrm{~b}$ & 1,1 & $\mathrm{~d}$ & 14,5 & $\mathrm{~b}$ & 11,8 & $\mathrm{~b}$ & 2,5 & $\mathrm{c}$ & 0,9 & $\mathrm{~b}$ & 23,3 & $\mathrm{~b}$ & 5,6 & $\mathrm{~b}$ & 38,4 & B \\
\hline Calc. & 9,8 & $\mathrm{c}$ & 0,9 & $\mathrm{~d}$ & 8,1 & $\mathrm{c}$ & 15,3 & $\mathrm{a}$ & 2,7 & $\mathrm{c}$ & 0,4 & $\mathrm{c}$ & 20,2 & $\mathrm{~b}$ & 5,8 & $\mathrm{~b}$ & 52,2 & A \\
\hline Test. & 8,7 & $\mathrm{c}$ & 1,0 & $\mathrm{~d}$ & 9,5 & $\mathrm{c}$ & 10,8 & & 2,1 & $\mathrm{c}$ & 0,3 & $\mathrm{c}$ & 20,0 & $\mathrm{~b}$ & 4,9 & $\mathrm{~b}$ & 34,6 & $\mathrm{~B}$ \\
\hline CV $(\%)$ & 12,9 & & 16 & & 8,23 & & 16,14 & & 16 & & 26, & & 23,1 & & 14,0 & & 20,3 & \\
\hline
\end{tabular}

${ }^{1}$ As média seguidas da mesma letra nas colunas, pertencem ao mesmo grupo, pelo teste de Scott-Knott, a 5\% de probabilidade.

Cerne, Lavras, v. 17, n. 4, p. 453-463, out./dez. 2011 
A omissão de calcário, Ca e micronutrientes favoreceu a absorção de K. Segundo Faquin (2005), concentrações elevadas de $\mathrm{Ca}$ e $\mathrm{Mg}$ reduzem a absorção de K por inibição competitiva. O K foi o segundo nutriente mais absorvido pelas plantas de cedro australiano. No tratamento 1, seu teor $\left(14,20 \mathrm{~g} \mathrm{~kg}^{-1}\right)$ foi superior ao encontrado por Souza et al. (2006), de 1,32 $\mathrm{g} \mathrm{kg}^{-1}$ em ipêroxo (Tabebuia impetiginosa).

$\mathrm{O}$ teor de $\mathrm{Ca}$ foi maior nos tratamentos com omissão de K, N, S e P. O maior teor de Ca, no tratamento com a omissão de $\mathrm{K}$, pode estar associado à inibição competitiva existente entre o $\mathrm{K}$ e o $\mathrm{Ca}$ no processo de absorção (MALAVOLTA, 1976; MALAVOLTA et al., 1997). De acordo com Malavolta e Violante Netto (1989), é quase impossível obter-se teores elevados dos dois nutrientes na MSPA, então, a ausência de K pode ter favorecido a absorção de $\mathrm{Ca}$ nas mudas de cedro australiano.

$\mathrm{O}$ teor de $\mathrm{Mg}$ foi maior no tratamento com a omissão de $\mathrm{K}$ e menor nos demais tratamentos (Tabela 5), possivelmente, pela redução da inibição competitiva entre o K e o Mg (FAQUIM, 2005). Verificou-se, ainda, um efeito de diluição, observando-se, no tratamento completo, um teor de $\mathrm{Mg}$ um pouco acima da testemunha. Contudo, esse teor $\left(2,5 \mathrm{~g} \mathrm{~kg}^{-1}\right)$ é superior aos teores encontrados por Braga et al. (1995), na MSPA de plantas de Acacia mangium $\left(1,3 \mathrm{~g} \mathrm{~kg}^{-1}\right)$, mas é inferior ao teor do tratamento completo da quaresmeira (Tibouchina granulosa) $(3,2$ $\mathrm{g} \mathrm{kg}^{-1}$ ) e ao da peroba-rosa (Aspidosperma polyneuron) $\left(4,0 \mathrm{~g} \mathrm{~kg}^{-1}\right)$.

As plantas de cedro australiano apresentaram teor de $\mathrm{S}$ inferior sob sua omissão (Tabela 5), sendo 2,2 vezes menor que o teor no tratamento completo 1 . Resultado semelhante foi encontrado por Venturin et al. (2005), para a candeia (Eremanthus erythropappus (DC) McLeish), cujo teor foi 2,5 vezes menor que o do tratamento completo aplicado para essa espécie.

Os tratamentos com omissão de N, P, K, -calc, compl. 1, compl. 2 e $\mathrm{Cu}$ apresentaram plantas com os maiores teores de B na MSPA $(30,5 ; 34,1 ; 28,4 ; 26,6 ; 32,6$; 27,5 e $27,6 \mathrm{mg} \mathrm{kg}^{-1}$, respectivamente), em virtude do efeito de concentração causado pelas suas menores produções.

A absorção de $\mathrm{Zn}$ foi favorecida pelas omissões de $\mathrm{P}, \mathrm{K}$ e $\mathrm{Cu}$, em razão da não ocorrência, no caso do $\mathrm{P}$, de inibição não competitiva. Entretanto, na omissão de $P$ e K, a planta se desenvolveu menos, ocorrendo um efeito de concentração no teor de $\mathrm{Zn}$, de modo semelhante ao que ocorreu para o B.

Cerne, Lavras, v. 17, n. 4, p. 453-463, out./dez. 2011
$\mathrm{O}$ teor de $\mathrm{Cu}$ foi maior no tratamento com omissão de $\mathrm{Zn}$, não diferindo estatisticamente dos tratamentos completo 1, omissão de calcário, omissão de $\mathrm{K}, \mathrm{S}$, micronutrientes, $\mathrm{Ca}, \mathrm{B}$ e $\mathrm{Cu}$. No tratamento com omissão de $\mathrm{Zn}$, pode ter havido uma diminuição da inibição competitiva entre o $\mathrm{Zn}$ e o $\mathrm{Cu}$, favorecendo a absorção desse último.

Observa-se que a omissão de $\mathrm{S}$ provocou alterações na nutrição da planta, resultado semelhante aos obtidos por Braga et al. (1995), para a pereira (Platycyamus regnellii), peroba-rosa (Aspidosperma polyneuron), Acacia mangium, e quaresmeira (Tibouchina granulosa).

A absorção de $\mathrm{N}$ foi favorecida pela omissão de $\mathrm{S}$ e K. A omissão de $\mathrm{S}$ favoreceu também a absorção de $\mathrm{P}$. Contrariamente ao encontrado por Venturin et al. (1999), os tratamentos sob omissão de $\mathrm{Ca}, \mathrm{S}$ e de $\mathrm{P}$ não resultaram em maiores teores de K na MSPA. Já, para o Ca, a omissão de $\mathrm{S}$ favoreceu a absorção daquele nutriente. $\mathrm{O} \mathrm{Mg}$ alcançou maiores teores na omissão de K. A omissão de K, Ca e Mg não afetou a absorção de S.

\subsection{Alterações morfológicas decorrentes da falta de nutrientes}

As plantas submetidas aos tratamentos com omissão de nutrientes apresentaram sintomas visuais de deficiência, quando o nível exigido para o seu crescimento vegetal ficou abaixo do considerado suficiente. $\mathrm{O}$ efeito da omissão de um nutriente resulta no decréscimo de sua concentração na planta e seus sintomas tornam-se claramente visíveis, quando uma deficiência nutricional é aguda e a taxa de crescimento e a produção são afetadas (MARSCHNER, 1995).

Os primeiros sintomas de deficiência foram constatados já na primeira avaliação (30 dias após o transplantio) e apareceram na seguinte ordem: S, calcário e N.

\subsubsection{Deficiência de nitrogênio (N)}

$\mathrm{O}$ sintoma característico de deficiência de $\mathrm{N}$ nas plantas é uma clorose (amarelecimento) que começa nas folhas mais velhas, com as folhas mais novas permanecendo verdes, inicialmente, em função da redistribuição (FAQUIN, 2005). As plantas de cedro australiano no tratamento em que houve omissão de $\mathrm{N}$ apresentaram tamanho reduzido e menor número de folhas. Verificou-se que as folhas mais velhas perderam gradualmente a coloração verde para uma tonalidade verde-pálida, a qual progrediu para um amarelo intenso, 
distribuindo-se uniformemente no limbo, pecíolo e nervuras. A coloração amarelada está associada à menor produção de clorofila e com modificações na forma dos cloroplastos (MALAVOLTA et al., 1997). De acordo com Malavolta (2006), o N é o nutriente mais exigido, sendo constituinte de uma série de compostos com inúmeras funções na planta. Observou-se, também, redução generalizada no diâmetro do caule e no desenvolvimento de raízes. As mesmas observações foram descritas por Batista et al. (2003) ao estudarem sintomas de deficiência nutricionais em gravioleira.

\subsubsection{Deficiência de fósforo $(\mathrm{P})$}

Em razão dos papéis do $\mathrm{P}$ na vida da planta, participando da síntese e degradação de macromoléculas (FAQUIN, 2005), a sua carência se refletiu no menor crescimento das plantas, com menor número de folhas e a raiz principal mais longa e com poucas raízes laterais. As folhas das plantas deficientes em $P$ apresentaram tonalidade amarelada, com pouco brilho e, posteriormente, arroxeadas. Os mesmos sintomas foram relatados por Malavolta et al. (1997) como indicadores da deficiência de P.

\subsubsection{Deficiência de potássio (K)}

As plantas deficientes em $\mathrm{K}$ apresentaram-se de tamanho reduzido, a exceção da MSR que teve seu valor estatisticamente entre os valores maiores. Os sintomas de deficiência de potássio foram observados inicialmente nas folhas mais velhas. A partir do ápice dessas folhas, verificou-se clorose marginal, avançando em direção à parte central, por entre as nervuras, inicialmente de coloração verde-amarela para, posteriormente, marrom, como consequência da necrose. Segundo Faquin (2005), pela mobilidade do K nos tecidos, os sintomas de deficiência ocorrem em primeiro lugar nas folhas mais velhas, com uma clorose seguida de necrose nas pontas e margens das folhas; nas regiões lesadas há acúmulo de putrescina.

\subsubsection{Deficiência de cálcio $(\mathrm{Ca})$}

A omissão de cálcio acarretou anormalidades visíveis nas folhas mais novas. Estas se mostraram recurvadas para baixo e com surgimento de clorose seguida por necrose nas pontas das folhas. As plantas com deficiência de cálcio também apresentaram um menor número de folhas e as raízes mostraram-se menos desenvolvidas e mais espessas, com poucas raízes laterais e de coloração escura. Pela baixa translocação do cálcio na planta, os sintomas de deficiência do nutriente ocorrem nos pontos de crescimento da parte aérea e da raiz, sendo as regiões de maior expansão celular as mais afetadas pela deficiência do nutriente (FAQUIN, 2005). Os sintomas observados por Marques et al. (2004), ao estudarem o efeito da omissão de nutrientes em paricá (Schizolobium amazonicum), foram semelhantes aos descritos nesse estudo.

\subsubsection{Deficiência de magnésio $(\mathrm{Mg})$}

Ao contrário do que ocorre com o Ca e semelhante ao $\mathrm{K}$, o Mg é móvel no floema, por isso, os sintomas manifestam-se primeiro nas folhas mais velhas, como clorose internerval. Percebeu-se, também, que a altura da planta foi inferior com a omissão do nutriente. Os sintomas observados foram semelhantes aos descritos por Malavolta et al. (1997). Segundo esses autores, a clorose observada é decorrente da redução do teor de clorofila. A função mais conhecida do $\mathrm{Mg}$ é compor a molécula de clorofila (VITTI et al., 2006). Assim, a sua deficiência reduziria a fotossíntese, causando a clorose, o que afetaria o crescimento da plantas, corroborando com o ocorrido nessa pesquisa,à exceção da MSR.

\subsubsection{Deficiência de enxofre (S)}

As plantas com carência de $\mathrm{S}$ apresentaram coloração verde nas folhas novas, em um tom mais claro em relação às folhas do tratamento completo. $\mathrm{O}$ caule mostrou-se mais delgado e com crescimento em altura reduzido. Observou-se que o sistema radicular foi bastante afetado, pela omissão do nutriente. Os resultados obtidos neste trabalho são similares aos constatados por Souza et al. (2006), estudando a adubação mineral do ipê-roxo (Tabebuia impetiginosa). Entretanto, Locatelli et al. (2007) verificaram que a omissão do nutriente, em plantas de cedro rosa (Cedrela odorata L.), pouco afetou o tamanho das plantas e o desenvolvimento da raiz.

\section{CONCLUSÕES}

Nas condições do presente estudo, pode-se concluir que:

- o nível de fertilidade do solo na condição natural limitou o desenvolvimento das plantas de cedro australiano;

- a omissão de nutrientes ocasionou efeito no crescimento do cedro australiano, sugerindo maior exigência nutricional da espécie;

- a sequência de exigência nutricional apresentada pelas mudas de cedro australiano, considerando a produção de matéria seca da parte aérea, em ordem decrescente, é: $\mathrm{P}>\mathrm{N}>\mathrm{S}>\mathrm{Ca}>\mathrm{K}>\mathrm{Mg}>\mathrm{Cu}$;

Cerne, Lavras, v. 17, n. 4, p. 453-463, out./dez. 2011 
- a omissão de B e Zn não afetou o crescimento das plantas de cedro australiano;

- a aplicação de calcário é essencial para o desenvolvimento de mudas do cedro australiano;

- o aparecimento dos primeiros sintomas de deficiência nutricional foi decorrente da omissão de $\mathrm{S}$, calcário e N.

\section{AGRADECIMENTOS}

Os autores agradecem à Associação dos Produtores Florestais do Sudoeste de Minas Gerais-APFLOR, ao Instituto Estadual de Florestas de Minas Gerais-IEF e à Bela Vista Florestal, pelo apoio financeiro para a realização do presente trabalho.

\section{REFERÊNCIAS}

BATISTA, M. M. F.; VIÉGAS, I. J. M.; FRAZÃO, D. A. C.; THOMAZ, M. A. A.; SILVA, R. C. L. Efeito da omissão de macronutrientes no crescimento, nos sintomas de deficiências nutricionais e na composição mineral em gravioleiras (Annona muricata). Revista Brasileira de Fruticultura, Jaboticabal, v. 25, n. 2, p. 315-318, 2003.

BLAKE, G. R.; HARTGE, K. H. Bulk density. In: KLUTE, A. (Ed.). Methods of soil analysis: physical and mineralogical methods. 2. ed. Madison: American Society of Agronomy, 1986. p. 363-375.

BRAGA, F. de A.; VALE, F. R. do; VENTURIN, N.; AUBERT, E.; LOPES, G. de A. Exigências nutricionais de quatro espécies florestais. Revista Árvore, Viçosa, v. 19, n. 1, p. 18-31, jan./mar. 1995.

CHAMINADE, R. Recherches sur fertilité et la fertilisation des sols dans les regions tropicales. Agronomie, Paris, v. 27, n. 9, p. 8891-8904, 1972.

CLARKSON, D. T. Adaptações morfológicas e fisiológicas das plantas a ambientes de baixa fertilidade. In: SIMPÓSIO SOBRE RECICLAGEM DE NUTRIENTES E AGRICULTURA DE BAIXOS INSUMOS NOS TRÓPICOS, 1984, Ilhéus. Anais... Ilhéus: CEPLAC/SBCS, 1985. p. 45-75.

EMPRESA BRASILEIRA DE PESQUISA AGROPECUÁRIA. Manual de métodos de análise de solo. 2. ed. Rio de Janeiro: CNPS, 1997. 212 p.

Cerne, Lavras, v. 17, n. 4, p. 453-463, out./dez. 2011
FAQUIN, V. Nutrição mineral de plantas. Lavras: UFLA/ FAEPE, 2005. 183 p.

FERREIRA, D. F. Análises estatísticas por meio do Sisvar (Sistema para análise de variância) para Windows 4.0. In: REUNIÃO ANUAL DA REGIÃO BRASILEIRA DA SOCIEDADE INTERNACIONAL DE BIOMETRIA, 45., 2000, São Carlos. Anais... São Carlos: UFSCar, 2000. p. 255-258.

FREIRE, J. C.; RIBEIRO, M. A. V.; BAHIA, V. G.; LOPES, A. S.; AQUINO, L. H. Resposta do milho cultivado em casa de vegetação a níveis de água no solo de região de Lavras, MG. Revista Brasileira de Ciência do Solo, Campinas, v. 4, n. 1, p. 5-8, jan./abr. 1980 .

LOCATELLI, M.; MACÊDO, R. S.; VIEIRA, A. H. Avaliação da altura e diâmetro de mudas de cedro rosa (Cedrela odorata L.) submetidas a diferentes deficiências nutricionais. Revista Brasileira de Biociências, Porto Alegre, v. 5, n. 2, p. 645-647, jul. 2007. Suplemento.

MALAVOLTA, E. Elementos de nutrição mineral de plantas. São Paulo: Ceres, 1980. 251 p.

MALAVOLTA, E. Manual de química agrícola. São Paulo: Agronômica Ceres, 1976. 528 p.

MALAVOLTA, E. Manual de nutrição mineral de plantas. São Paulo: Agronômica Ceres, 2006. 638 p.

MALAVOLTA, E.; VIOLANTE NETTO, A. Nutrição mineral, calagem, gessagem e adubação dos citros. Piracicaba: POTAFOS, 1989. 153 p.

MALAVOLTA, E.; VITTI, G. C.; OLIVEIRA, S. A. Avaliação do estado nutricional das plantas: princípios e aplicações. 2. ed. Piracicaba: POTAFOS, 1997. 319 p.

MARQUES, T. C. L. L. S. M.; CARVALHO, J. G.; LACERDA, M. P. C.; MOTA, P. E. F. Crescimento inicial do paricá (Schizolobium amazonicum) sob omissão de nutrientes e de sódio em solução nutritiva. Cerne, Lavras, v. 10, n. 2, p. 184-195, 2004

MARSCHNER, H. Mineral nutrition of higher plants. London: Academic, 1995.890 p.

PINHEIRO, A. L.; LANI, L. L.; COUTO, L. Cultura do cedro australiano para produção de madeira serrada. Viçosa, MG: UFV, 2003. 42 p. 
PINHEIRO, A. L.; RAMALHO, R. S.; BARREIROS, H. S. Árvores exóticas em Viçosa: II., Toona ciliata M. Roem. var. australis (F. V. M.) C. DC. (MELIACEAE). Revista Ceres, Viçosa, v. 41, n. 234, p. 103-112, 1994.

RAIJ, B. van; QUAGGIO, J. A.; SILVA, N. M. da. Extraction of phosphorus, potassium, calcium, and magnesium from soils by an ion-exchange resin procedure. Communications in Soil Science and Plant Analysis, New York, v. 17, n. 5, p. 547-566, 1986.

SANCHES, P. A. Suelos de los trópicos: características y manejo. San José: IICA, 1981. 660 p.

SILVA, E. B.; GONÇALVES, N. P.; PINHO, P. J. Limitações nutricionais para crescimento de mudas de umbuzeiro em Latossolo Vermelho distrófico no norte de Minas. Acta Scientiarum Agronomy, Maringá, v. 27, n. 1, p. 55-59, 2005.

SILVA, W. G.; TUCCI, C. A.; HARA, F. A. S.; SANTOS, R. A. C. Efeito de micronutrientes sobre o crescimento de mudas de mogno (Swietenia macrophylla King) em Latossolo Amarelo. Acta Amazonica, Manaus, v. 37, n. 3, p. 371-376, 2007.

SOUZA, P. A.; VENTURIN, N.; MACEDO, R. L. G. Adubação mineral do Ipê-Roxo. Ciência Florestal, Santa Maria, v. 16, n. 3, p. 261-270, 2006.

VENTURIN, N.; MENDONÇA, A. V. R.; NOGUEIRA, F. D. Exigências nutricionais de Myracroduon urundeuva Fr. All (Aroeira do sertão). Cerne, Lavras, v. 5, n. 2, p. 65-75, 1999.

VENTURIN, N.; SOUZA, P. A.; MACEDO, R. L. G.; NOGUEIRA, F. D. Adubação mineral da candeia (Eremanthus erythropappus (DC) McLeish). Floresta, Curitiba, v. 35, n. 2 , p. 211-219, 2005.

VITTI, G. C.; LIMA, E.; CICARONE, F. Cálcio, magnésio e enxofre. In: FERNANDES, M. S. (Ed.). Nutrição mineral de plantas. Viçosa, MG: Sociedade Brasileira de Ciência do Solo, 2006. p. 299-325.

Cerne, Lavras, v. 17, n. 4, p. 453-463, out./dez. 2011 
